\title{
As indicações de cesáreas no Brasil: uma revisão de literatura integrativa
}

\author{
The indications for cesarean sections in Brazil: a review of the integrative literature
}

Las indicaciones de cesáreas en Brasil: una revisión de literatura integrativa

Aline Palermo da Silva ${ }^{1 *}$, Rhaissa Toledo Romero ${ }^{1}$, Adriana Bragantine ${ }^{1}$, Andrielle Aparecida Diniz Martins Barbieri ${ }^{1}$, Milena Torres Guilhem Lago ${ }^{1}$.

\section{RESUMO}

Objetivo: Analisar quais são as indicações de cesáreas no Brasil. Métodos: Uma revisão de literatura integrativa, utilizando-se a Biblioteca Virtual em Saúde (BVS), onde 11 artigos foram selecionados de acordo com os critérios de inclusão e exclusão pré-estabelecidos. Resultados: Constatou-se que ao longo da última década as indicações mais predominantes para a cesariana no país foram os pedidos maternos pelo procedimento e as indicações médicas não claras, seguidas de outras indicações médicas, sendo: laqueadura, iteratividade, desproporção céfalo-pélvica, obesidade materna, apresentação pélvica, sofrimento fetal agudo, gestação prolongada, falha de indução e síndromes hipertensivas. Conclusão: Concluiu-se que a maior parte das indicações de cesáreas não possuía caráter absoluto, cabendo a avaliação individualizada dos casos. O estudo surpreendeu ao evidenciar de forma prevalente os pedidos maternos e as indicações médicas não claras, suscitando importantes discussões sobre esses pontos, enfatiza-se a importância do prénatal, a inserção do enfermeiro nesse contexto, a indispensabilidade de amplo conhecimento, a disposição médica para aplicar diferentes manejos e, acima de tudo, a mudança de postura por parte destes baseada na melhor evidência científica e não na ação mais cômoda, retomando a cultura de que o foco é garantir a segurança fetal e materna.

Descritores: Cesárea, Enfermagem, Obstetrícia, Saúde da mulher.

\begin{abstract}
Objective: To analyze the indications for cesarean section in Brazil. Methods: A review of the integrative literature, using the Virtual Health Library (VHL), where 11 articles were selected according to the preestablished inclusion and exclusion criteria. Results: It was observed that during the last decade the most prevalent indications for cesarean section in the country were the maternal requests by the procedure and the unclear medical indications, followed by other medical indications: itchiness, iterativity, pelvic cephalic abruption, hypertensive syndromes, induction failure, acute fetal distress, pelvic presentation, post-datitis and maternal obesity. Conclusion: It was concluded that the majority of cesarean indications are not absolute, and the obstetrician is responsible for the individualized evaluation of the cases. The study was surprising to highlight prevalent maternal orders and unclear medical indications, raising important discussions about these points, emphasizing the importance of prenatal care, the insertion of nurses in this context, the indispensability of broad knowledge, the provision medical care to apply different treatments and, above all, the change of posture on the part of these based on the best scientific evidence and not on the more practical and comfortable action, resuming the culture that the focus is to guarantee the fetal and maternal security.
\end{abstract}

Descriptores: Cesarean section, Nursing, Obstetrics, Women's health.

${ }^{1}$ Centro Universitário Filadélfia - UniFil. Londrina/PR. *E-mail: aline.pallermo@hotmail.com

SUBMETIDO EM: $3 / 2019$

ACEITO EM: 4/2019

PUBLICADO EM: 6/2019

REAS/EJCH | Vol.Sup.24 | e624 | DOI: https://doi.org/10.25248/reas.e624.2019 Página 1 de 9 


\section{RESUMEN}

Objetivo: Analizar cuáles son las indicaciones de cesáreas en Brasil. Métodos: Una revisión de literatura integrativa, utilizando la Biblioteca Virtual en Salud (BVS), donde 11 artículos fueron seleccionados de acuerdo con los criterios de inclusión y exclusión preestablecidos. Resultados: Se constató que a lo largo de la última década las indicaciones más predominantes para la cesárea en el país fueron las solicitudes maternas por el procedimiento y las indicaciones médicas no claras, seguidas de otras indicaciones médicas, siendo: laqueadura, iteratividad, desproporción cefalo-pélvica, obesidad materna, presentación pélvica, sufrimiento fetal agudo, gestación prolongada, falla de inducción y síndromes hipertensivos. Conclusión: Se concluyó que la mayor parte de las indicaciones de cesáreas no poseía carácter absoluto, cabiendo la evaluación individualizada de los casos. El estudio sorprendió al evidenciar de forma prevalente las solicitudes maternas y las indicaciones médicas no claras, suscitando importantes discusiones sobre esos puntos, se enfatiza la importancia del prenatal, la inserción del enfermero en ese contexto, la indispensable de amplio conocimiento, la disposición y que, por encima de todo, el cambio de postura por parte de éstos basada en la mejor evidencia científica y no en la acción más cómoda, retomando la cultura de que el foco es garantizar la seguridad fetal y materna.

Descriptores: Cesárea, Enfermería, Obstetricia, Salud de la mujer.

\section{INTRODUÇÃO}

A cesariana é uma das cirurgias mais comuns no mundo atual. Sabe-se que a operação, em seus primórdios, foi o recurso encontrado para salvar as vidas dos fetos vivos cujas mães estavam mortas ou prestes a morrer, porém, com o surgimento de novas técnicas cirúrgicas, da anestesia, dos recursos farmacológicos e a melhora nos métodos de antissepsia, a cesárea deixou de significar obrigatoriamente a morte da mãe, tornando-se uma alternativa relativamente segura para casos específicos em que a gestante e o feto encontrem-se em situações de risco (ALONSO BD, 2015).

Segundo Ribeiro (2016), no Brasil a cesárea também é um dos procedimentos cirúrgicos mais comuns. 0 país passa por uma verdadeira epidemia de cesarianas, sendo atribuído a ele o título de líder mundial dessas operações, passando de $4 \%$ na década de 70 para uma taxa atual de $55 \%$ do total de partos, muito díspar quando comparada com a taxa de $20 \%$ da Europa e de $30 \%$ do Estados Unidos e, também, muito preocupante tendo em vista que desde 1985 a comunidade médica internacional considera que a taxa ideal de cesáreas fique entre 10\% e 15\% (BRASIL 2016a; ORGANIZAÇÃO MUNDIAL DE SAÚDE (OMS), 2015).

Ao comparar as vias de parto no Brasil, entre os anos 2008 e 2016, percebe-se que apenas no ano de 2008 o parto natural foi predominante à cesariana, com uma diferença de quase 100 mil casos e, em todos os anos subsequentes, a cesárea prevaleceu com diferenças importantes variando entre 300 mil e 400 mil casos, isto mesmo com a implantação de importantes planos de ação, como é o caso do projeto Parto Adequado, lançado em 2015 e que em 18 meses evitou a realização de 10 mil operações desnecessárias, evidenciando resultados transformacionais, mas reforçando a necessidade de empenho contínuo para impactar no atual cenário nacional (ANS 2016; BRASIL 2016a).

Estudos mostram que os serviços privados e a rede suplementar se apresentam como importantes responsáveis nos dados alarmantes do país. Segundo informações mais recentes divulgadas por eles, a taxa de partos cesáreos é de 40\% na rede pública, chegando a 84\% nos serviços supramencionados (ANS 2016).

É difícil definir as razões do aumento dos casos de cesáreas no Brasil nas últimas décadas, mas Amorim MMR et al (2010) dizem que várias hipóteses podem ser levantadas para explicar a preferência pela cesariana que existe atualmente, essas causas incluem a maneira como a assistência ao nascimento é organizada no país; ainda bastante centrada na atuação individual dos profissionais em contraposição à abordagem multidisciplinar, as características socioculturais, a qualidade dos serviços que assistem os nascimentos e as características da assistência pré-natal; que comumente deixa de preparar adequadamente as mulheres para o parto e nascimento.

REAS/EJCH | Vol.Sup.24 | e624 | DOI: https://doi.org/10.25248/reas.e624.2019 Página 2 de 9 
A OMS (2015) afirma que quando realizadas por motivos médicos, as cesarianas podem reduzir a mortalidade e morbidade materna e perinatal, porém não existem evidências de que fazer cesáreas em mulheres ou bebês que não necessitem dessa cirurgia traga benefícios, pois assim como qualquer cirurgia, uma cesárea acarreta riscos imediatos e à longo prazo.

Torna-se nítido que a operação cesariana traz benefícios a gestantes e crianças quando sua indicação é bem determinada, todavia há a necessidade de evitar-se a cesariana desnecessária. Montenegro $\mathrm{C}$ e Rezende J (2014) lembram que quando comparadas ao parto normal espontâneo, as cesáreas eletivas sem indicação médica estão associadas a maior morbidade e mortalidade materna e infantil, além de consequente elevação de gastos para o sistema de saúde do país.

Diante do que foi explanado, surgiu o interesse pela temática por considerar que o momento do nascimento instiga questões sobre o processo do parto e via de parto e também por compreenderas implicações de morbidade e mortalidade materna e infantil quando a indicação da cesariana não é bem determinada, ou seja, quando não possui uma real indicação, sendo necessário, por parte de todos os profissionais de saúde, incluindo o enfermeiro, a busca pelo entendimento mais amplo sobre as indicações de cesariana para que possíveis soluções possam ser pensadas. Sendo assim, o presente estudo teve como objetivo analisar quais são as indicações de cesáreas no Brasil. Para isto, o estudo teve a seguinte questão norteadora: o que está publicado na literatura científica, nos últimos 10 anos, quanto as indicações de cesáreas no Brasil?

\section{MÉTODO}

O presente estudo trata-se de uma revisão de literatura integrativa. A busca na literatura foi realizada na Biblioteca Virtual em Saúde (BVS), famosa por contemplar importantes bases de dados, no mês de abril de 2018. Utilizou-se quatro Descritores em Ciências da Saúde (DeCS), sendo eles: cesárea, enfermagem, obstetrícia e saúde da mulher.

O primeiro momento de investigação iniciou-se com o principal descritor; cesárea, sendo expostos 43.145 materiais no acervo. Foram, então, utilizados cinco critérios de inclusão pré-estabelecidos para melhor condução do trabalho. Apenas materiais no formato de artigos científicos, disponíveis na íntegra, publicados nos últimos 10 anos; sendo de 2008 a 2017, somente no idioma português, tratando do Brasil como país de assunto, resultando em 146 pesquisas. Continuando, com um critério de exclusão, os artigos em formato de revisão de literatura foram descartados, restando 122 potenciais materiais. Posteriormente, uma leitura exploratória dos títulos foi realizada e os artigos repetidos ou fora da temática descartados, prosseguiu-se com uma leitura seletiva, aprofundando a investigação com leitura dos resumos, aqueles que não correspondiam ao tema em investigação também foram excluídos, assim, nove artigos foram salvos.

Foram utilizados os descritores integrados através do operador boleano "AND", ainda respeitando as normas de inclusão e de exclusão mencionadas, apenas dois novos itens foram selecionados, desta forma, resultando em 11 artigos científicos. A análise dos dados ocorreu de forma sintetizada, organizada e crítica por meio da utilização de um instrumento de coleta de dados, contemplando as seguintes informações: título, autores, formação do principal autor, ano de publicação, periódico, objetivos, abordagem metodológica e resultados principais. Esta etapa possibilitou a interpretação dos dados, garantindo a obtenção de respostas ao problema da pesquisa. Após a conclusão das quatro etapas anteriores, definiu-se a amostra desse trabalho, composta pelos 11 artigos supramencionados. Por conseguinte, os resultados foram expostos, discutidos e apresentados através de uma categoria, apresentada a seguir: as indicações de cesáreas no Brasil na última década.

\section{RESULTADOS E DISCUSSÕES}

Conforme demonstra o quadro 1 abaixo, foram apresentados os 11 artigos científicos que contemplaram os critérios de inclusão e exclusão pré-definidos nessa pesquisa. Identificou-se que $63,6 \%$ dos artigos analisados possuíam como autor principal enfermeiros (B, D, E, G, H, I, J), sendo que no restante da amostra, sendo $36,3 \%$, eram profissionais médicos ( $A, C, F, K)$. 
No que tange o período de publicação estudado, foram encontrados artigos nos anos 2009, 2010, 2011, 2014, 2015, 2016 e 2017, ressalta-se que os anos que mais se destacaram por conterem maior número de publicações, igualmente, foram 2009, 2010, 2011 e 2014, totalizando 72,7\% da amostra e, nos anos de 2008, 2012 e 2013 não foram encontradas pesquisas sobre a temática.

Importante revelar que a maioria dos artigos, sendo $81,9 \%$, estava relacionada a uma abordagem quantitativa ( $A, B, C, D, E, F, H, J, K$ ), apenas $18,1 \%$ utilizava abordagem qualitativa (G, I).

Também vale salientar que os artigos estavam contemplados em 11 periódicos distintos, sendo eles: Biblioteca de Saúde Pública (E), Cad. de Saúde Pública do Rio de Janeiro (B, H), Diagnóstico e Tratamento (A), Rev. Bioética (C), Rev. Brasileira de Ginecologia e Obstetrícia (F), Rev. da Escola de Enfermagem da USP (J), Rev. da Faculdade de Ciências Médicas de Sorocaba (K), Rev. de Pesquisa: Cuidado é Fundamental Online (G), Rev. Saúde Pública do Rio de Janeiro (D) e Rev. de Saúde Coletiva do Rio de Janeiro (I).

Quadro 1 - Distribuição por códigos dos artigos científicos publicados no Brasil, entre os anos de 2008 a 2017, utilizados durante a revisão de literatura integrativa, Londrina, 2018.

\begin{tabular}{|c|c|c|c|c|c|}
\hline CÓDIGO & TÍTULO & AUTOR & ANO & POPULAÇÃO & OBJETIVO \\
\hline A & $\begin{array}{l}\text { Dados epidemiológicos, } \\
\text { evidências e reflexões } \\
\text { sobre a indicação de } \\
\text { cesariana no Brasil }\end{array}$ & Sass et al. & 2009 & $\begin{array}{lr}\text { Os } & \text { dados } \\
\text { disponíveis } & \text { no } \\
\text { Sistema } & \text { de } \\
\text { Informações } & \text { dos } \\
\text { Nascidos } & \text { Vivos } \\
\text { (Sinasc; } & \text { tabnet) } \\
\text { relativos } & \text { à } \\
\text { assistência } & \text { ao parto } \\
\text { no Brasil. }\end{array}$ & $\begin{array}{l}\text { Evidenciar e refletir sobre as } \\
\text { indicações de cesárea no } \\
\text { Brasil. }\end{array}$ \\
\hline B & $\begin{array}{l}\text { Aspectos relacionados à } \\
\text { escolha do tipo de parto: } \\
\text { um estudo comparativo } \\
\text { entre uma maternidade } \\
\text { pública e outra privada, } \\
\text { em São Luís, Maranhão, } \\
\text { Brasil }\end{array}$ & $\begin{array}{l}\text { Mandarino } \\
\text { NR et al. }\end{array}$ & 2009 & 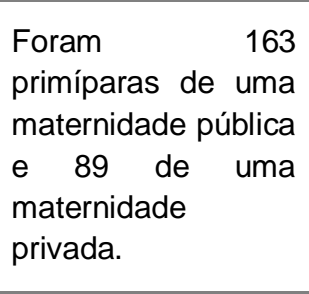 & $\begin{array}{l}\text { Analisar aspectos } \\
\text { relacionados à escolha do tipo } \\
\text { de parto em uma maternidade } \\
\text { do serviço público e outra do } \\
\text { serviço privado, em São Luís, } \\
\text { Maranhão, Brasil. }\end{array}$ \\
\hline C & $\begin{array}{lr}\text { Atitudes } & \text { dos } \\
\text { profissionais de } & \text { destetrícia em relação à } \\
\text { obstarto } \\
\text { escolha da via de parto } \\
\text { em Porto Velho, } \\
\text { Rondônia, Brasil }\end{array}$ & $\begin{array}{l}\text { Ferrari } \mathrm{J} \text { et } \\
\text { al. }\end{array}$ & 2010 & $\begin{array}{l}\text { Todos os } \\
\text { Ginecologistas } \\
\text { Obstétricos da } \\
\text { Região de Porto } \\
\text { Velho, Rondônia. }\end{array}$ & $\begin{array}{l}\text { Objetivou levantar a opinião, } \\
\text { as condutas médicas e as } \\
\text { atitudes éticas dos } \\
\text { profissionais da obstetrícia de } \\
\text { Porto Velho, Rondônia, em } \\
\text { relação à indicação de } \\
\text { cesarianas. }\end{array}$ \\
\hline D & $\begin{array}{l}\text { Fatores associados à } \\
\text { realização de cesariana } \\
\text { em hospitais brasileiros }\end{array}$ & $\begin{array}{l}\text { Pádua KS } \\
\text { et al. }\end{array}$ & 2010 & $\begin{array}{l}\text { Mulheres que } \\
\text { tiveram parto entre } \\
\text { setembro/2004 e } \\
\text { março/2005. }\end{array}$ & $\begin{array}{llll}\text { Avaliar a } & \text { prevalência de } \\
\text { cesariana } & \text { em } & \text { hospitais } \\
\text { brasileiros. } & & \end{array}$ \\
\hline $\mathbf{E}$ & $\begin{array}{l}\text { Fatores associados à } \\
\text { preferência por } \\
\text { cesariana em uma } \\
\text { amostra representativa } \\
\text { de primíparas na Região } \\
\text { Sul do Brasil, } 2011\end{array}$ & $\begin{array}{l}\text { Soalheiro } \\
\text { LC et al. }\end{array}$ & 2011 & $\begin{array}{lr}\text { Foram } & 1.904 \\
\text { primíparas } & \text { da } \\
\text { Região Sul do } \\
\text { Brasil, cuja média de } \\
\text { idade foi de } 26 \text { anos. }\end{array}$ & $\begin{array}{lrr}\text { Traçar } & 0 & \text { perfil } \\
\text { sociodemográico } & \text { e } \\
\text { reprodutivo de puérperas } \\
\text { primíparas da Região Sul do } \\
\text { Brasil, segundo sua } \\
\text { preferência pela cesariana, no } \\
\text { início da gravidez. }\end{array}$ \\
\hline $\mathbf{F}$ & $\begin{array}{l}\text { Estratégias dirigidas aos } \\
\text { profissionais para a } \\
\text { redução das cesáreas } \\
\text { desnecessárias no Brasil }\end{array}$ & $\begin{array}{l}\text { Haddad } \\
\text { SEMT et al. }\end{array}$ & 2011 & $\begin{array}{l}\text { Profissionais da } \\
\text { saúde (médicos e } \\
\text { enfermeiros) do } \\
\text { Brasil. }\end{array}$ & $\begin{array}{l}\text { Reduzir as cesáreas } \\
\text { desnecessárias no Brasil, por } \\
\text { meio de estratégias dirigidas } \\
\text { aos profissionais de saúde. }\end{array}$ \\
\hline
\end{tabular}




\begin{tabular}{|c|c|c|c|c|c|}
\hline G & $\begin{array}{l}\text { A cultura interferindo no } \\
\text { desejo sobre o tipo de } \\
\text { parto }\end{array}$ & $\begin{array}{l}\text { Pimenta LF } \\
\text { et al. }\end{array}$ & 2014 & $\begin{array}{l}\text { Foram } 8 \text { mulheres } \\
\text { em idade fértil, com } \\
\text { história pregressa } \\
\text { de parto vaginal ou } \\
\text { cesariano, que não } \\
\text { estavam no período } \\
\text { gravídico-puerperal. }\end{array}$ & $\begin{array}{l}\text { Compreender de que forma a } \\
\text { cultura influencia no processo } \\
\text { de parturição da mulher. }\end{array}$ \\
\hline H & $\begin{array}{l}\text { Fatores Associados à } \\
\text { cesariana entre } \\
\text { primíparas adolescentes } \\
\text { no Brasil, 2011-2012 }\end{array}$ & $\begin{array}{l}\text { Gama SGN } \\
\text { et al. }\end{array}$ & 2014 & $\begin{array}{l}\text { Foram } 90 \text { puérperas } \\
\text { no período de } 2011 \\
\text { e } 2012 \text { em hospitais } \\
\text { mistos. }\end{array}$ & $\begin{array}{l}\text { Verificar fator associados a } \\
\text { cesariana entre primíparas } \\
\text { adolescentes no Brasil. }\end{array}$ \\
\hline I & $\begin{array}{l}\text { A normalização da } \\
\text { cesárea como modo de } \\
\text { nascer: cultura material } \\
\text { do parto em } \\
\text { maternidades privadas } \\
\text { no Sudeste do Brasil }\end{array}$ & $\begin{array}{l}\text { Nakano et } \\
\text { al. }\end{array}$ & 2015 & $\begin{array}{l}\text { Foram } 33 \text { mulheres } \\
\text { com idade entre } 18 \\
\text { e } 35 \text { anos, que } \\
\text { realizaram cesárea } \\
\text { em } 2011 \text { no setor } \\
\text { privado de } \\
\text { assistência à saúde } \\
\text { na cidade do Rio de } \\
\text { Janeiro ou São } \\
\text { Paulo. }\end{array}$ & $\begin{array}{l}\text { Analisar relatos de mulheres } \\
\text { urbanas que realizaram } \\
\text { cesáreas em maternidades } \\
\text { privadas. }\end{array}$ \\
\hline $\mathbf{J}$ & $\begin{array}{l}\text { Fatores associados ao } \\
\text { parto cesárea nos } \\
\text { sistemas público e } \\
\text { privado de atenção à } \\
\text { saúde }\end{array}$ & $\begin{array}{l}\text { Oliveira RR } \\
\text { et al. }\end{array}$ & 2016 & $\begin{array}{lr}\text { Foram } & 920 \\
\text { puérperas } & \text { do } \\
\text { município } & \text { de } \\
\text { Maringá/PR, sendo } & \\
485 \text { que realizaram } \\
\text { o parto pelo sistema } \\
\text { público de saúde e } \\
435 \text { pelo sistema } \\
\text { privado, entre } \\
\text { outubro de } 2013 \text { e } \\
\text { fevereiro de } 2014 \text {. }\end{array}$ & $\begin{array}{l}\text { Identificar fatores associados à } \\
\text { cesárea entre residentes do } \\
\text { município de Maringá-PR, } \\
\text { segundo fonte de } \\
\text { financiamento do parto. }\end{array}$ \\
\hline K & $\begin{array}{l}\text { Indicações de partos } \\
\text { cesáreos em hospitais } \\
\text { de atendimento } \\
\text { ao Sistema Único de } \\
\text { Saúde: baixo e alto } \\
\text { riscos }\end{array}$ & $\begin{array}{l}\text { Novo JLVG } \\
\text { et al. }\end{array}$ & 2017 & $\begin{array}{l}\text { Foram } 150 \\
\text { pacientes } \\
\text { de baixo risco } \\
\text { atendidas no HSL e } \\
150 \text { de alto risco no } \\
\text { CHS, por intermédio } \\
\text { do Sistema Único de } \\
\text { Saúde (SUS), } \\
\text { identificadas pelo } \\
\text { Serviço de Arquivo } \\
\text { Médico e Estatístico } \\
\text { (SAME) de cada } \\
\text { hospital, todas elas } \\
\text { submetidas a partos } \\
\text { cesáreos no período } \\
\text { de } 01 \text { de março de } \\
2014 \text { a } 01 \text { de } \\
\text { março de } 2015 \text {. }\end{array}$ & $\begin{array}{l}\text { Investigar as } \\
\text { indicações dos partos } \\
\text { cesáreos no Hospital Santa } \\
\text { Lucinda } \\
\text { (HSL) - de baixo risco - e no } \\
\text { Conjunto Hospitalar de } \\
\text { Sorocaba } \\
\text { (CHS). }\end{array}$ \\
\hline
\end{tabular}

Fonte: próprios autores (2018).

\section{As indicações de cesáreas no Brasil na última década}

Foi exposto em seis pesquisas, sendo as de Mandarino NR et al. (2009), Sass et al. (2009), Haddad SEMT et al. (2011), Soalheiro LC et al. (2011), Gama SGN et al. (2014) e Oliveira RR et al. (2016), como principal 
responsável pelas altas taxas de cesarianas, o pedido materno pelo procedimento, que ocorre, predominantemente, no formato de cesárea eletiva, mas também no intraparto. Importante salientar que o Conselho Federal de Medicina (CFM) (2016) afirma que é direito da gestante, de risco habitual, a partir da 39 a semana de gestação, optar pela realização de cesariana.

Discutindo tal achado, Pelloso SM et al (2000), Dias MAB et al. (2008), Silva SPC et al (2014) e Santana FA et al (2015) apontam que esse pedido ocorre, basicamente, por medo da dor do trabalho de parto, medo do parto normal comprometer a anatomia vaginal e portanto a vida sexual, superestimação da segurança que o uso da tecnologia fornece com relação à saúde do bebê e da própria mulher e subestimação dos riscos da cesárea, desconhecimento dos benefícios do parto normal, experiências prévias com o parto, história e influência familiar; com destaque para a figura da mãe, influência de profissionais da saúde e, de forma enfática, falta de informações durante a atenção pré-natal.

Destacando esse último ponto, Brasil (2016b) ressalta que se deve fornecer informações para as gestantes durante toda assistência pré-natal baseadas em evidências científicas atualizadas e envolver a família durante esse processo, pois essa prática é tida como uma ferramenta poderosa de empoderamento capaz de remodelar a atual cultura cesarista encontrada no país e, além disso, também é recomendado que a gestante converse sobre sua preferência com outros profissionais, incluindo enfermeiras(os) obstétricas(os), pois Silvestre DR et al. (2014) apontam que quando há inserção de enfermeiros nesse processo, a contribuição para a redução das cesáreas sem reais indicações é ainda mais efetiva.

A indicação médica para a cesárea, foi encontrada também em seis artigos, mantendo o formato tanto de cesárea eletiva, quanto no intraparto e, sabe-se, segundo a OMS (2015), que essa indicação deveria corresponder a no máximo 15\% dos partos e estar relacionada aos critérios absolutos de recomendação, porém as justificativas não ficaram claras na maioria dos casos encontrados (FERRARI J et al, 2010; HADDAD SEMT et al. 2011; SOALHEIRO LC et al., 2011; GAMA SGN et al., 2014; PIMENTA LF et al., 2014; NAKANO et al., 2015).

Ao pensar nas duas indicações supracitadas, é interessante observar que a literatura evidencia que a preferência de $70 \%$ a $80 \%$ das mulheres que sofreram cesárea nos setores privado e público antes do nascimento, era pelo parto vaginal, mas em algum momento da gestação ou do próprio parto, elas optaram pela cesariana (PERPÉTUO IHO, et al 1998; D'ORSI E et al., 2006; DIAS MAB et al., 2008).

Contra essa preferência, Salgado (2012) sugere que há uma frequente persuasão por parte dos profissionais médicos para suas pacientes aceitarem uma cesariana programada por razões que não existem ou que não justificam este procedimento e MARTINS COSTA CSRA et al (2005) dizem que esse procedimento agendado coloca em evidência um modelo de conveniência que deixa muito claro o conflito de interesses por trás da indicação, já que esta permite ao obstetra organizar sua agenda pessoal e profissional e aumentar o número de pacientes atendidas, o que faz refletir sobre a prevalência de indicações médicas sem justificativas claras.

A realização da laqueadura foi o indicativo para cesárea encontrado em três artigos, sendo por pedido materno ou indicação médica (MANDARINO NR et al. 2009; PÁDUA KS et al. 2010; PIMENTA LF et al. 2014). Porém, para Brasil (1996; 2015; 2016b) é recomendado que o modo de nascimento não seja determinado em função da realização da ligadura tubária e que a laqueadura não seja realizada no momento do parto, exceto nos casos de comprovada necessidade por operações cesarianas sucessivas anteriores, risco de vida materno em uma futura gestação e risco de vida para um futuro concepto, obedecendo os critérios de ser maior de 25 anos ou com mais de dois filhos vivos. Preconizam, que para isto, o planejamento familiar deve acontecer antes e durante o pré-natal e ter acompanhamento por equipe multidisciplinar a fim de desencorajar a esterilização fora das circunstâncias recomendadas (BRASIL, 1996; 2015; 2016b).

A iteratividade, significado dado a mulher que já teve três ou mais cesáreas, caracterizou a quarta indicação de cesárea mais encontrada (OLIVEIRA RR et al. 2016; NOVO JLVG et al. 2017). É recomendado que as mulheres com operações cesarianas prévias sejam esclarecidas de que há um aumento no risco de ruptura uterina com o parto vaginal. Sabe-se que esse risco é, a princípio, baixo, porém aumenta à medida 
que aumenta o número de operações cesarianas prévias, sendo consenso que a operação cesariana é recomendada para mulheres com três ou mais operações cesarianas prévias, que a conduta deve ser individualizada para mulheres com duas operações cesarianas prévias ou com intervalo entre partos inferior a 15 meses e, que na ausência de outras contraindicações, é recomendado encorajar a mulher com uma operação cesariana prévia a tentativa de parto vaginal (BRASIL, 2015; 2016b).

As pesquisas de Pádua KS et al. (2010) e Novo JLVG et al. (2017) abordaram a indicação de cesárea por desproporção céfalo-pélvico (DCP), sendo que na primeira a indicação foi fora de trabalho de parto e na segunda intraparto. Amorim MMR et al (2010) dizem que a DCP frequentemente é diagnosticada de forma inadequada, pois o diagnóstico só pode ser realizado durante o trabalho de parto, baseado nos dados de sua evolução, conforme registro no partograma, não devendo se supor uma DCP, mesmo quando se conhece os principais fatores de riscos para desproporção, que são peso fetal acima de $3.000 \mathrm{~g}$, obesidade materna, nuliparidade e pelvimetria alterada. Aliás, inexiste evidência que apoie o uso da pelvimetria clínica ou por radiografia para predizer a ocorrência de falha de progressão do trabalho de parto, não devendo mais ser mais estimulada e usada para decisão da via do nascimento (BRASIL, 2015, 2016b).

A obesidade materna foi um indicador de cesárea encontrado nos artigos de Pádua KS et al. (2010) e Oliveira RR et al. (2016). Segundo Silva JC et al. (2014), comparadas às gestantes com peso normal, pacientes obesas apresentam chances maiores do desenvolvimento de diabetes gestacional, síndrome hipertensiva, hemorragia de grande porte no momento do parto, macrossomia fetal e recém-nascidos com Ápgar baixo no primeiro minuto, assim, está associada com maior necessidade de cuidados durante o prénatal e parto pelas chances de intercorrências maternas e neonatais, sendo comum a operação cesariana.

Corroborando, Brasil (2015; 2016b) trazem uma análise que comparou cesariana programada e parto vaginal planejado, onde não se evidenciou diferenças significativas, exceto pela distócia de ombro que foi maior naquelas que tiveram partos normais, por conseguinte, a operação cesariana não é recomendada como forma rotineira de nascimento de feto de mulheres obesas, devendo a decisão ser individualizada.

Novo JLVG et al. (2017) trazem que apresentação pélvica também é uma indicação de cesárea encontrada no país. Brasil $(2015 ; 2016 b)$ reforçam que um parto pélvico vaginal é viável, mas que a assistência deve, impreterivelmente, ser realizada por profissionais experientes e a que a mulher seja informada sobre o maior risco de morbidade e mortalidade perinatal e neonatal.

Além disso, reforçam que como primeira escolha, na ausência de contraindicações e com consentimento da mulher, deve ser feita a manobra de versão cefálica externa a partir de 36 semanas de idade gestacional, mas nas situações nas quais a versão cefálica externa estiver contraindicada ou não tiver sucesso, a operação cesariana pode ser sugerida a partir de 39 semanas de idade gestacional caso haja recusa da mulher pelo parto vaginal ou insegurança da equipe para condução do trabalho de parto e, preferencialmente, após o início do trabalho de parto (BRASIL, 2015; 2016b).

No artigo de Novo JLVG et al. (2017) o sofrimento fetal agudo (SFA) foi apontado como indicação de cesárea também. O SFA é conceitualmente a asfixia persistente e progressiva cuja gravidade, se não revertida, leva ao esgotamento dos mecanismos compensatórios fetais, resultando em morte celular neuronal, podendo ser reconhecido de forma indireta por alterações nas atividades biofísicas fetais, principalmente em sua frequência cardíaca, identificada pela ausculta ou traçado cardiotocográfico (CTG) (MONTENEGRO C e REZENDE J 2014).

Osana e Reis (2012) dizem que geralmente o SFA ocorre durante o trabalho de parto e que o tratamento consiste na ressuscitação fetal intraútero, que muitas das vezes não é capaz de reverter ou reduzir o quadro de hipóxia, seguida de finalização do parto pela via mais rápida, seja pela via abdominal (cesariana de urgência), seja pela via vaginal (nos casos em que o parto é iminente e/ou é possível aplicação do fórceps) se a ausculta ou CTG persistirem suspeitos. A cesárea indicada por gravidez prolongada (GP) foi encontrada em um artigo, sendo o de Novo et al. (2017). É unânime a definição de que a GP é a gestação com duração igual ou superior a 42 semanas completas ou com mais de 294 dias, contados a partir do primeiro dia do último período menstrual (OMS, 2006). 
Matthes ACS (2010) lembra que a GP tem uma incidência que varia de 3 a $14 \%$ das gestações, dependendo se foi contada a partir da data da última menstruação (DUM) ou pela ultrassonografia (USG) e, também, mostra que atentar-se para o acompanhamento da idade gestacional (IG) por um dos dois métodos é fundamental, pois, o risco de morte perinatal, especialmente de natimortalidade pré-parto, aumenta com o avançar da idade gestacional, além de riscos maternos como rotura cervical, hemorragia pós parto, infecção puerperal, dano perineal grave e o dobro de risco de cesárea. Por isso, a recomendação atual é de que quando o parto não ocorre até a $41^{\underline{a}}$ semana a conduta de induzir o trabalho de parto em todas as gestantes com 41 semanas de gravidez é preferível à avaliação seriada do bem-estar fetal, pois se observou menor risco de morte neonatal e perinatal e menor chance de cesariana no grupo submetido à indução do parto com 41 semanas (BRASIL, 2012a).

Novo JLVG et al. (2017) trazem que a falha de indução foi uma outra indicação de cesárea. A indução do parto consiste em estimular artificialmente as contrações uterinas coordenadas e efetivas antes de seu início espontâneo, levando ao desencadeamento do trabalho de parto em mulheres a partir da $22^{\mathrm{a}}$ semana de gravidez (SOUZA ASR et al., 2010). As principais indicações para indução do trabalho de parto, abrange desde indicações eletivas, como gestantes na $41^{\text {a }}$ semana de gravidez sem doença obstétrica, até quando a interrupção da gravidez se faz de fato necessária devido a doenças maternas ou fetais, que fazem com que o prolongamento da gestação ocasione risco de morte materno ou fetal (SOUZA ASR et al., 2010). Para Sampaio ZS et al. (2004), na maioria das condições clínicas representativas de indicação para indução do parto, a cérvice desfavorável é encontrada, o que pode frequentemente acarretar falha da indução e consequente aumento da incidência de cesarianas.

As síndromes hipertensivas foram indicativas de cesárea no estudo de Novo JLVG et al. (2017). Conforme lembra Brasil (2012b), o parto vaginal é preferível à cesariana para mulheres com síndromes hipertensivas, desse modo evitando o estresse adicional de uma cirurgia em uma situação de alterações fisiológicas múltiplas. Medidas paliativas por várias horas não aumentam o risco materno se realizadas de forma apropriada e a indução do parto deve ser realizada de forma intensiva assim que a decisão para a interrupção for tomada, sabe-se que a abordagem intensiva para a indução inclui um ponto final claro para o parto, de cerca de 24 horas após o início do processo e, que, se o parto vaginal não puder ser efetuado dentro de um período razoável de tempo, deve-se realizar a cesariana (BRASIL 2012b).

\section{CONCLUSÃO}

Indo contra a meta internacional que considera a taxa ideal de cesáreas entre $10 \%$ e $15 \%$, o Brasil encontra-se com uma taxa atual de $55 \%$ do total de partos. Concluiu-se com esse estudo que ao longo da última década as indicações mais predominantes para a cesariana no Brasil foram os pedidos maternos pelo procedimento e as indicações médicas não claras. Sobre essas indicações enfáticas, é importante perceber a importância do pré-natal, a inserção do enfermeiro nesse contexto, a indispensabilidade de amplo conhecimento, a disposição médica para aplicar diferentes manejos e, acima de tudo, a mudança de postura por parte destes baseada na melhor evidência científica e não na ação mais cômoda, retomando a cultura de que o foco é garantir a segurança fetal e materna.

\section{REFERÊNCIAS}

1. AGÊNCIA NACIONAL DE SAÚDE SUPLEMENTAR. Sociedade Beneficente Israelita Brasileira. Hospital Albert Einstein. Institute for Healthcare Improvement. Nova organização do cuidado ao parto e nascimento para melhores resultados de saúde projeto parto adequado - fase 1. Rio de Janeiro. 2016. 49 p.

2. ALONSO BD. Fatores associados à cesariana segundo fonte de financiamento da região sudeste: estudo transversal a partir dos dados de pesquisa "nascer no Brasil: inquérito nacional sobre parto e nascimento". 2015. 68 f. Dissertação (Mestrado em Saúde Pública) - Faculdade de Saúde Pública da Universidade de São Paulo, São Paulo, 2015.

3. AMORIM MMR, et al. Indicações de cesariana baseadas em evidências: parte I. FEMINA, Rio de Janeiro, v. 38, n. 8, p. 415422, ago. 2010

4. BRASIL. Presidência da República. Casa Civil Subchefia para Assuntos Jurídicos. Lei no 9.263, de 12 de janeiro de 1996. Trata do planejamento familiar.

5. Ministério da Saúde. Cadernos de atenção básica: atenção ao pré-natal de baixo risco (CAB 32). Bras, $2012 a .320$ p. 
6. Ministério da Saúde. Secretaria de Atenção à Saúde. Departamento de Ações Programáticas Estratégicas. Gestação de alto risco: manual técnico. 5. ed. Brasília, 2012b. 302 p. (Série A. Normas e Manuais Técnicos).

7. Ministério da Saúde. Diretrizes de atenção à gestante: a operação cesariana, protocolo relatório de recomendação. Brasília, 2015. $101 \mathrm{p}$.

8. Ministério da Saúde. Informações de saúde: estatísticas vitais: tipos de parto. Brasília, 2016a.

9. Ministério da Saúde. Secretaria de Atenção à Saúde. Portaria № 306, de 28 de março de 2016. Aprova as Diretrizes de Atenção à Gestante: a operação cesariana.

10. DIAS MAB et al. Trajetória das mulheres na definição pelo parto cesáreo: estudo de caso em duas unidades do sistema de saúde suplementar do estado do Rio de Janeiro. Ciência \& Saúde Coletiva, Rio de Janeiro, v. 13, n. 5, 2008.

11. CONSELHO FEDERAL DE MEDICINA. Resolução no 2.144/2016, é ético o médico atender à vontade da gestante de realizar parto cesariano, garantida a autonomia do médico, da paciente e a segurança do binômio materno fetal. Brasília: CFM, 2016. 4 p.

12. D'ORSI E et al. Fatores associados à realização de cesáreas em uma maternidade pública do município do Rio de Janeiro, Brasil. Cad. Saúde Pública, Rio de Janeiro, v. 22, p. 2067-78, 2006.

13. FERRARI J, LIMA NM. Atitudes dos profissionais de obstetrícia em relação à escolha da via de parto em Porto Velho, Rondônia, Brasil. Revista Bioética, Brasília, v. 18, n. 3, p. 645-58, 2010.

14. GAMA SGN et al. Fatores associados à cesariana entre primíparas adolescentes no Brasil, 2011-2012. Cad. Saúde Pública, Rio de Janeiro, v. 30, n. 1, p. 117-127, 2014.

15. HADDAD SEMT, CECECATTI JG. Estratégias dirigidas aos profissionais para a redução das cesáreas desnecessárias no Brasil. Rev. Bras. Ginecol. Obstet., Rio de Janeiro, v. 33, n. 5, p. 252-262, maio. 2011.

16. MANDARINO NR et al. Aspectos relacionados à escolha do tipo de parto: um estudo comparativo entre uma maternidade pública e outra privada, em São Luís, Maranhão, Brasil. Cad. Saúde Pública, Rio de Janeiro, v. 25, n. 7, p.1587-1596, jul.2009.

17. MARTINS CSRA, LOPES GJ. A questão das cesarianas. Rev. Bras. Ginecol. Obstet., Rio de Janeiro, v. 27, n. 4, 2005.

18. MATTHES ÂCS. Gravidez prolongada: subsídios da literatura médica para uma defesa. FEMINA, Rio de Janeiro, v. 38 , n. 8, ago. 2010.

19. MENDES KDS, et al. Revisão integrativa: método de pesquisa para a incorporação de evidências na saúde e na enfermagem. Texto Contexto Enferm., Florianópolis, v. 17, n. 4, p. 758-64, out.-dez. 2008.

20. MONTENEGRO C, REZENDE J. Obstetrícia fundamental. 13. ed. Rio de Janeiro: Guanabara Koogan, 2014. 764 p.

21. NAKANO AR; BONAN, Claudia; TEIXEIRA, Luiz Antônio. A normalização da cesárea como modo de nascer: cultura material do parto em maternidades privadas no Sudeste do Brasil. Physis, Rio de Janeiro, v. 25, n. 3, p. 885-904, set. 2015.

22. NOVO JLVG et al. Indicações de partos cesáreos em hospitais de atendimento ao Sistema Único de Saúde: baixo e alto riscos. Revista da Faculdade de Ciências Médicas de Sorocaba, Sorocaba, v. 19, n. 2, p. 67-71, jun. 2017.

23. ORGANIZAÇÃO MUNDIAL DA SAÚDE. Declaração da OMS sobre taxas de cesáreas. Genebra: OMS, 2015. 8 p.

24. OLIVEIRA RR et. al. Fatores associados ao parto cesárea nos sistemas público e privado de atenção à saúde. Rev. Esc. Enferm. USP, São Paulo, v. 50, n 4, p. 734-741. 2016.

25. PÁDUA KS et al. Fatores associados à realização de cesariana em hospitais brasileiros. Rev. Saúde Pública, São Paulo, v. 44, n. 1, p. 70-79, fev. 2010.

26. PELLOSO SM et al. Opção ou imposição: motivos da escolha da cesárea. R. Arq. Ciênc. Saúde Unipar, Umuarama, v. 4, n. 1, p. 3-8, jan./abr., 2000.

27. PERPÉTUO IHO, et al. Parto cesáreo: uma análise da perspectiva das mulheres de Belo Horizonte. In: ENCONTRO NACIONAL DE ESTUDOS POPULACIONAIS DA ASSOCIAÇÃO BRASILEIRA DE ESTUDOS POPULACIONAIS, 10., 1998, Caxambu. Anais... Caxambu: Associação Brasileira de Estudos Populacionais, 1998, p. 95-119.

28. PIMENTA LF et al. A cultura interferindo no desejo sobre o tipo de parto. Rev. Pesqui. Cuid. Fundam. (Online), Porto Alegre, v. 6 , n. 3, p. 987-997, jul.-set. 2014.

29. RIBEIRO LB. Nascer em Belo Horizonte: cesarianas desnecessárias e prematuridade. 2016. 115 f. Dissertação (Mestrado em Enfermagem) - Escola de Enfermagem da Universidade Federal de Minas Gerais, Belo Horizonte, 2016.

30. SALGADO HO. A experiência da cesárea indesejada: perspectivas das mulheres sobre decisões e suas implicações no parto e nascimento. 2012. 157 f. Dissertação (Mestrado em Saúde Pública) - Faculdade de Saúde Pública da Universidade de São Paulo, São Paulo, 2012.

31. SAMAPIO ZS et al. Fatores associados ao parto vaginal em gestantes de alto risco submetidas à indução do parto com Misoprostol. Rev. Bras. Ginecol. Obstet., São Paulo, v. 26, n. 1, p. 21-29, 2004.

32. SANTANA FA, et al. Fatores que influenciam a gestante na escolha do tipo de parto. Rev. Fac. Ciênc. Méd. Sorocaba, v.17, n.3, p. $123-127,2015$.

33. SASS N, HWANG SM. Dados epidemiológicos, evidências e reflexões sobre a indicação de cesariana no Brasil. Diagn. Tratamento, São Paulo, v. 14, n. 4, p. 133-7, 2009.

34. SILVA JC et al. Obesidade durante a gravidez: resultados adversos da gestação e do parto. Rev. Bras. Ginecol. Obstet., São Paulo, v. 36, n. 11, p. 509-13, 2014.

35. SILVA SPC e; PRATES, Renata de Carvalho Gomes; CAMPELO, Bruna Queiroz Armentano. Parto normal ou cesariana: fatores que influenciam na escolha da gestante. Rev. Enferm. UFSM, Santa Maria, v. 4, n. 1, p. 1-9, 2014.

36. SILVESTRE DR et al. Via de parto orientada no pré-natal e a escolha da enfermeira no seu próprio parto. Revista de Enfermagem online, Recife, v. 8, n, 12, p. 4230-6, dez. 2014.

37. SOALHEIRO LC. Fatores associados à preferência por cesariana em uma amostra representativa de primíparas na Região Sul do Brasil, 2011. 85 f. Dissertação (Mestrado em Saúde Pública) - Escola Nacional de Saúde Pública, Rio de Janeiro, 2011.

38. SOUZA ASR et al. Indução do trabalho de parto: conceitos e particularidades. FEMINA, Rio de Janeiro, v. 38, n. 4, p. 185194, abr. 2010.

39. WORLD HEALTH ORGANIZATION. International statistical classification of diseases and related health problems: tenth revision. Geneva: World Health Organization, 2006. 